\title{
Disparity in the spatial distribution of clinics within a metropolitan city
}

\author{
Kwang-Soo Lee \\ Department of Health Administration, College of Health Sciences, Yonsei University, Wonju, Gwangwondo, \\ South Korea
}

\begin{abstract}
A methodology for evaluating and understanding how healthcare agencies are distributed within a city is provided. The study undertaken analysed the disparity in the spatial distribution of clinics within the metropolitan city of Daejeon, South Korea. Address and specialty of clinics in use were collected from five public health centres in 2010. Buffer analysis, hot-spot analysis, and generalized linear models were applied to the data collected. Multivariate analysis was also conducted on data collected in 2008 from the annual records of five ward offices (mid-level city administration units) taking the lowest administrative level of the city (the dong) into account. Buffer analysis showed that numerous clinics were located near major roads, while the hot-spot analysis identified three areas with concentrations of clinics and one area with hardly any clinics. The results of the generalized linear models showed variations depending on the specialty of the clinics suggesting that their distribution differed depending on specialty. There are no current regulations in force governing clinic location. Policy makers should consider improving the clinic distribution taking their speciality into account.
\end{abstract}

Keywords: hot-spot analysis, clinics, geographical distribution, metropolitan city, South Korea.

\section{Introduction}

Spatial accessibility of primary care and preventive services influence their utilization (Comber et al., 2011). It follows that better accessibility of these services is one way of improving the performance of the healthcare system (Dulin et al., 2010). As the distance between primary care agencies and people's residences increases, the use of healthcare services significantly decreases (Tanser et al., 2006). Health screening rates vary according to the distance people are required to travel, e.g. $10 \mathrm{~km}$ increase in travel distance results in an odds ratio (OR) of 0.87 for breast cancer screening in Sweden (Maheswaran et al., 2006). Epstein (2001) argued that the availability of public ambulatory clinics provides better access to primary care for people with low-income and the elderly.

The spatial distribution of healthcare agencies in South Korea differs between urban and rural areas as more healthcare agencies are located in urban areas (Ministry of Health and Welfare, 2011). In 2010,

\footnotetext{
Corresponding author:

Kwang-Soo Lee

Department of Health Administration

College of Health Sciences, Yonsei University

Yonseidaegil 1, 220-710 Wonju, Gwangwondo, South Korea

Tel. +82 33760 2426; Fax + 82337602519

E-mail: planters@yonsei.ac.kr
}

$43.7 \%$ of all acute-care hospitals operated in seven metropolitan cities (Korean Hospital Association, 2010). In addition to the issue of the concentration of healthcare agencies in urban areas, their distribution within cities can also be problematic. Clinics perform a gatekeeper role for patient care and any spatial disparity in clinic distribution across a city limits this role.

An analysis of the spatial distribution of clinics within a city would provide basic information for evaluating the accessibility of primary care services to residents. Various spatial methods that have implications for analysing and understanding the issues in the healthcare sector have been proposed (Newacheck et al., 1996; Phillips et al., 2000; McLafferty, 2003; Alcaraz et al., 2009); these methods apply not only to hospitals but also to clinics (Guagliardo, 2004). If clinics were evenly distributed, people would not have to travel long distances, which would enhance the accessibility of care services (Comber et al., 2011).

Studies of the variation in the spatial distribution of primary care centres have been conducted based on the defined geographical service area or on the macro scale at the administrative district level (Parchman, 1995; Gravelle and Sutton, 2001; Mantzavinis et al., 2003; Theodorakis and Mantzavinis, 2005; Busato and Künzi, 2008). However, few empirical studies have analysed the spatial distribution of clinics within an urban area. In the current study, the actual locations of 
clinics in a selected, metropolitan city were recorded, and it was examined whether these clinics have an equal density across the city or if they vary by area. The spatial characteristics of the clinic locations was first investigated followed by a multiple regression analysis evaluating the relationship between the number of clinics and selected socioeconomic variables.

\section{Methods}

\section{Source and organization of the data}

This study was conducted in the city of Daejeon, which has a population slightly exceeding 1.5 million. It is one of six metropolitan cities in South Korea and all clinics operating in this city were included in the study. Data regarding the clinics were collected from the city's five public health centres in 2010. These centres collect and manage information related to all healthcare agencies within their area of responsibility. The number of clinics included in the analysis was 981 and their names, addresses and specialties were entered into our database. While the addresses identified the geographical location of the clinics, their names were used to classify the specialty, e.g. if the name of the clinic included the epithet "internal medicine", the specialty of clinic was designated as such in our records. If the name of a clinic did not contain information relating to its specialty, the head physician's specialty (obtained from the South Korean Health Insurance Review and Assessment Agency) was used.

The addresses of the 981 clinics were converted into $\mathrm{X}-\mathrm{Y}$ coordinates and entered into a geographical information system (GIS) by longitude and latitude. Then, a point layer containing the locations of the clinics was made based on a 2008 map obtained from the Statistical Geographical Information Service (SGIS) of the statistical centre in South Korea, i.e. Statistics Korea (http://kostat.go.kr/portal/english/index.action). This map provided the boundaries of administrative districts, location of roads, and the shapes and types of buildings. It included the smallest administrative districts (the dong), which is similar to the zip code system used in the United States of America (USA) and categorised the roads into three types: highway, major and minor arterial road. Smaller roads than the latter were not used in the analysis. Local regulations require that buildings cannot be erected closer than 12 or $70 \mathrm{~m}$ (measured from the centre of road) depending on the type of road.

\section{Spatial analysis and multiple regression}

The spatial data were entered into ArcMap, version 10 (ESRI; Redlands, CA, USA), which was then used for geocoding, and spatial analysis. The spatial clinics data were analysed in three steps. Initially, a buffer analysis method (Lee and Hong, 2010) was applied to analyse the general arrangement of clinics with respect to roads. It showed how many clinics were located within certain distances from the roads. A close location was assumed to increase awareness of the clinic and its accessibility to residents of the city (Vahidnia et al., 2009). A hot-spot analytical method (Getis and Ord, 1992) was then applied to evaluate the spatial density of clinics in the study area. Buffer and hot-spot analyses were conducted based on the actual positions of the clinics on the map. After testing the distribution of all clinics, two separate groups were developed to determine differences in spatial density according to clinic specialty. Group 1 included the specialties plastic surgery and dermatology, which had a high proportion of medical services that were not funded by the National Health Insurance programme. Group 2 included internal medicine and family medicine, which had a high proportion of medical services receiving support from this programme.

Multivariate analysis was conducted to test the relationship between the number of clinics and proxy variables representing the socioeconomic status of the residents. With the dependent variable being count data, linear regression would not be appropriate (Crawley, 2007). Therefore, generalized linear models (conducted with SPSS, version 19.0) were applied for the multivariate analysis. The variables used in this analysis were calculated with the dong level as base. The dependent variable was the number of clinics operating within the boundary of each dong. Total population, population over 65 years, number of businesses, number of employees and road area (a variable representing the range of the road system in each dong) were the independent variables as described by Lee and Hong (2010). Regression analysis was also conducted for the two clinic groups. The annual reports from the five ward offices (the next higher administrative level above the dong) provided data on the independent variables, except for the road area, which was generated from the road layer provided by the SGIS of Statistics Korea. A Kolmogorov-Smirnov test (Massey, 1951) revealed that the independent variables, except for the total population, were not normally distributed; hence these variables were transformed to their natural $\log$ to alleviate skewness (Carter et al., 1998). 


\section{Hot-spot analysis}

Hot-spot analysis was conducted based on the number of clinics per point identified under the address. Multiple clinics can operate at one point as they can be located in the same building and therefore use the same postal address. Thus, each point could represent a different number of clinics. This analysis, based on the Getis-Ord $G_{i}{ }^{*}$ statistic (Getis and Ord, 1992; Ord and Getis, 1994), distinguished spatial clusters of high concentrations of clinics (hot spots) from areas characterised by a dearth of clinics (cold spots). Z-scores and $\mathrm{p}$-values were calculated for each point and used to test for the difference between the actual number of clinics and the expected number in the different areas. A high, positive Z-score with a low p-value indicates a hot spot, while a negative Z-score with a low p-value indicates a cold spot. A score close to 0 indicates that there is no significant difference in clinic distribution in the area in question. The following equations were used for calculating these statistics:

$$
\begin{gathered}
G_{i}^{*}=\frac{\sum_{j=1}^{n} w_{i j} x_{j}-\bar{X} \sum_{j=1}^{n} w_{i j}}{s \sqrt{\frac{\left[n \sum_{j=1}^{n} w_{i j}^{2}-\left(\sum_{j=1}^{n} w_{i j}\right)^{2}\right]}{n-1}}} \\
\bar{X}=\frac{\sum_{j=1}^{n} x_{j}}{n} \\
s=\sqrt{\frac{\sum_{j=1}^{n} x_{j}^{2}}{n}-(\bar{X})^{2}}
\end{gathered}
$$

where $\chi_{j}$ is an attribute value for feature $j, \omega_{i, j}$ the spatial weight between features $i$ and $j$ and $n$ the total number of features.

\section{Results}

The outcome of the investigation provides quantitative information regarding the metropolitan distribution of clinics. Table 1 shows the number of clinics per point, which ranged between one and nine. In total, 692 points were identified, and approximately $79 \%$ of these represented only one clinic each. The average number of clinics per point was 1.41 with a standard deviation of 1.08 .
Table 1. The frequency of clinics per point.

\begin{tabular}{lcc}
\hline Number of clinics per point & Frequency & $\%$ \\
\hline 1 & 544 & 78.6 \\
2 & 90 & 13.0 \\
3 & 23 & 3.3 \\
4 & 16 & 2.3 \\
5 & 4 & 0.6 \\
6 & 6 & 0.9 \\
7 & 6 & 0.9 \\
8 & 1 & 0.1 \\
9 & 2 & 0.3 \\
\hline Total & 692 & 100.0 \\
\hline
\end{tabular}

The results of the buffer analysis revealed that the clinics had a clear spatial correlation to the roads and Table 2 shows that the number of clinics close to the road is large. When highways and major arterial roads were included, 342 clinics were within the $30 \mathrm{~m}$ buffer and 397 within the $50 \mathrm{~m}$ buffer. If the minor arterial roads were also included, the number of clinics within the buffers increased to 641 and 729 , respectively. The $50 \mathrm{~m}$ buffer, applied to highway and major arterial roads, encompassed about $40 \%$ of all clinics.

Figure 1 shows the distribution of all clinics in the study area (map A) and the Z-score calculated from the hot-spot analysis (map B). Many clinics were located in the centre of the city, and points that had a higher number of clinics were apparently situated in the central area. The Z-score calculated from the hot-spot analysis, based on all clinics, identified three hot spots and one cold spot.

Figure 2 shows detailed maps of the three hot spots identified in Fig. 2. Map B indicates the location with the highest density of clinics in the study area. Each point had an average of 2.5 clinics, and the number of clinics in the hot spot included $15.5 \%$ of all clinics. The hot spot in map C (comprising $1.4 \%$ of all clinics investigated), had an average of 2.4 clinics per point, while the hot spot in map D (comprising $3.9 \%$ of all clinics investigated), had an average of 2.3 clinics per point. The number of clinics in the three hot spot areas combined contained $20.8 \%$ of all clinics in the city, i.e. approximately one-fifth of all clinics within the city (204 out of the 981 clinics).

Figure 3 shows a detailed map of the cold spot. Here, most points had only one clinic, and the average number of clinics per point was 1.1. The total number of clinics in the cold spot area was approximately $1.8 \%$ of all (18 out of the 981 clinics). This region was part of the old downtown section of the city that was 
Table 2. Number of clinics within two different buffer distances.

\begin{tabular}{|c|c|c|c|c|c|c|}
\hline \multirow{2}{*}{$\begin{array}{l}\text { Road type } \\
\text { Highway + major arterial road }\end{array}$} & \multicolumn{2}{|c|}{$\begin{array}{l}\text { Clinics within } \\
30 \mathrm{~m} \text { of road* }\end{array}$} & \multicolumn{2}{|c|}{$\begin{array}{l}\text { Clinics within } \\
50 \mathrm{~m} \text { of road* }\end{array}$} & \multicolumn{2}{|c|}{ Total } \\
\hline & 342 & $(34.9 \%)$ & 397 & $(40.5 \%)$ & 981 & $(100.0 \%)$ \\
\hline Highway + major arterial road + minor arterial road & 641 & $(65.3 \%)$ & 729 & $(74.3 \%)$ & 981 & $(100.0 \%)$ \\
\hline
\end{tabular}

"Measured from the centre of the road.

built before the construction of the new urbanized area.

Table 3 shows the number of clinics in hot and cold spots identified by specialty. Among the clinics in hotspot areas, clinics specialising in plastic surgery and dermatology were present in a higher percentage than were other specialties. Nineteen clinics offering plastic surgery were located in hot-spot areas, constituting $73.1 \%$ of all plastic surgery clinics. Fifteen clinics offering dermatology were located in hot-spot areas, constituting $50.0 \%$ of all dermatology clinics. Specialties with a low percentage of clinics in both hot and cold spots were internal medicine ( 130 out of 158 clinics), family medicine (47 out of 56 clinics), paediatrics ( 70 out of 77 clinics), and orthopaedics (68 out of 75 clinics)

Hot-spot analysis was conducted for two separate clinic groups. Group 1 consisted of clinics specialising in plastic surgery and dermatology, and group 2 consisted of those specialising in internal medicine and family medicine (Fig. 4). The hot spots correspond to map B in Fig. 2. Among the 56 clinics offering plastic surgery and dermatology, 22 clinics $(39.3 \%)$ were located in hot spots. For the clinics offering internal medicine and family medicine, $21(9.8 \%)$ of 214 $(100.0 \%)$ clinics were located in hot spots. There was a higher density of plastic surgery and dermatology clinics in particular areas than there were internal medicine and family medicine clinics.

Table 4 shows the results of the generalized linear analysis. The Poisson regression model and negative Binomial model were fitted to the three types of clinic to be able to select the regression model with the best fit. It was found that the Poisson regression model showed the best fit to two of the groups (all clinics and clinics majoring in family medicine and internal medicine), while the negative Binomial model showed the best fit to the group of clinics majoring in plastic surgery and dermatology.

In model 1, which included all clinics, the population over 65 years, the number of businesses, and the number of employees contributed significantly to
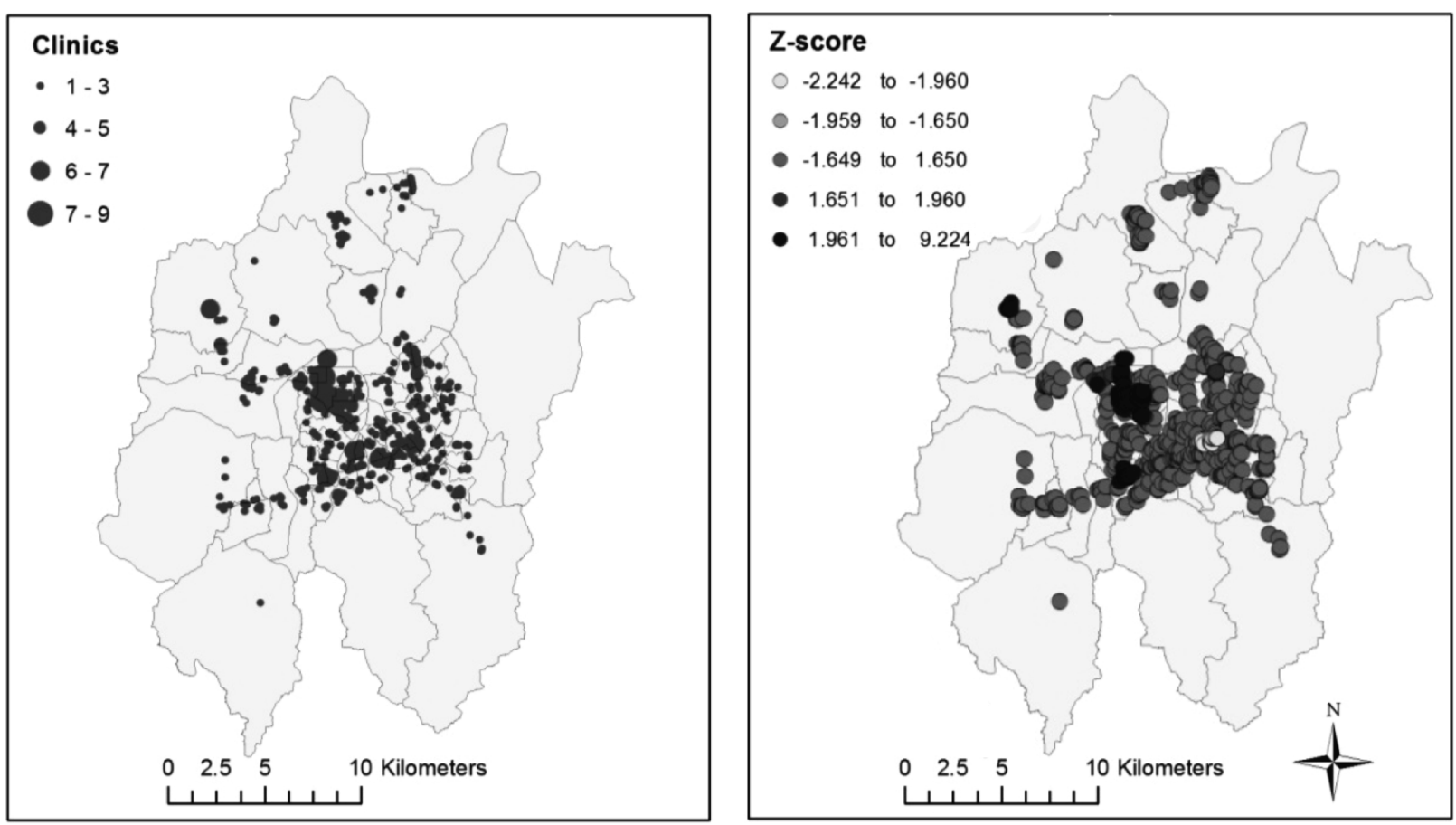

Fig. 1. Distribution of clinics (A) in Daejeon City and the Z-scores (B) according to hot-spot analysis. 


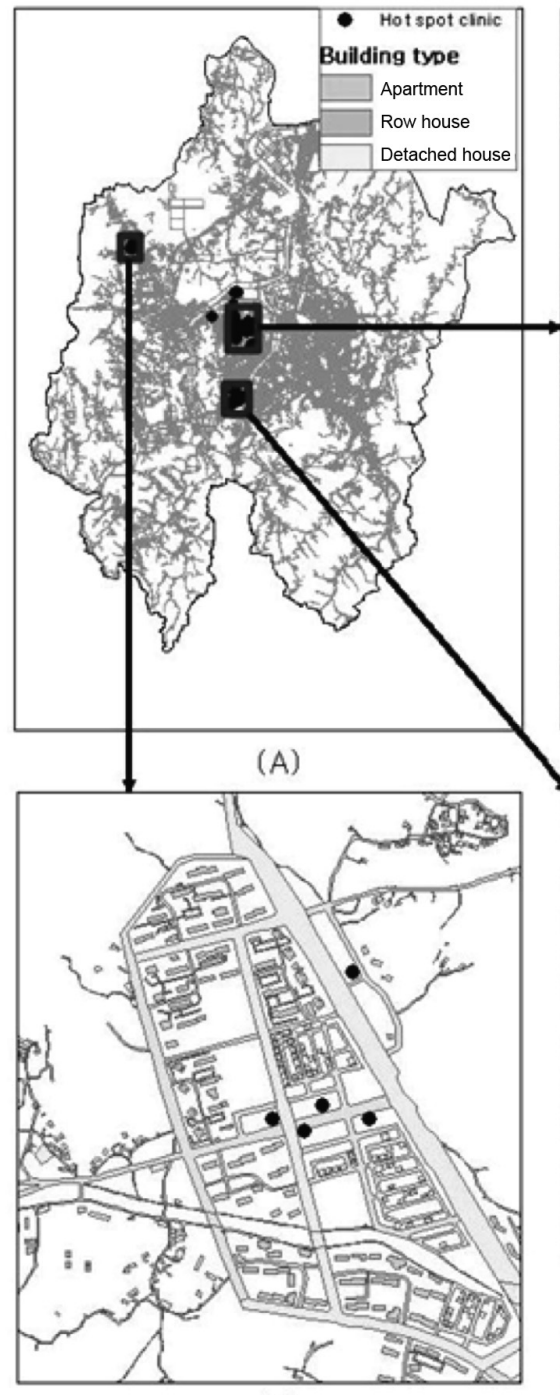

(C)

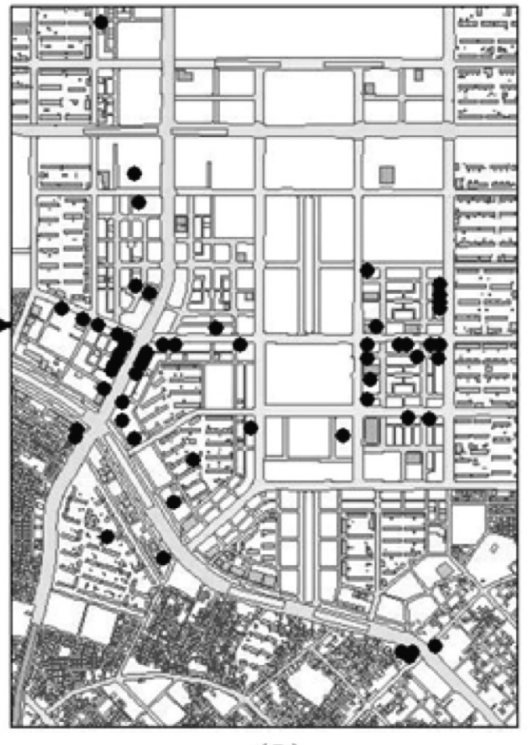

(B)

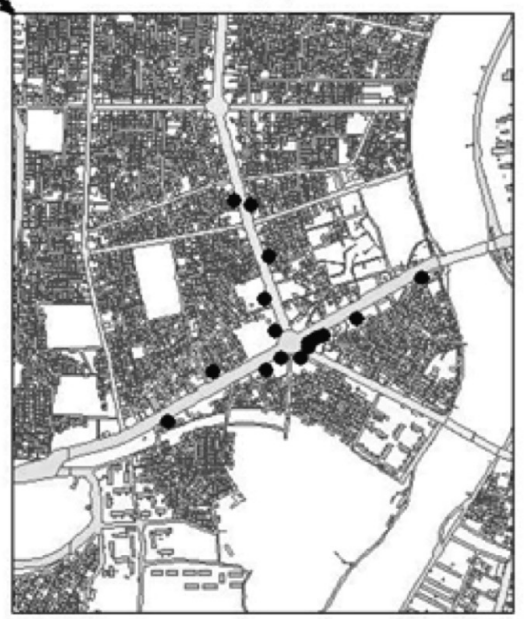

(D)

Fig. 2. Hot-spot areas with respect to clinics in Daejeon, South Korea.
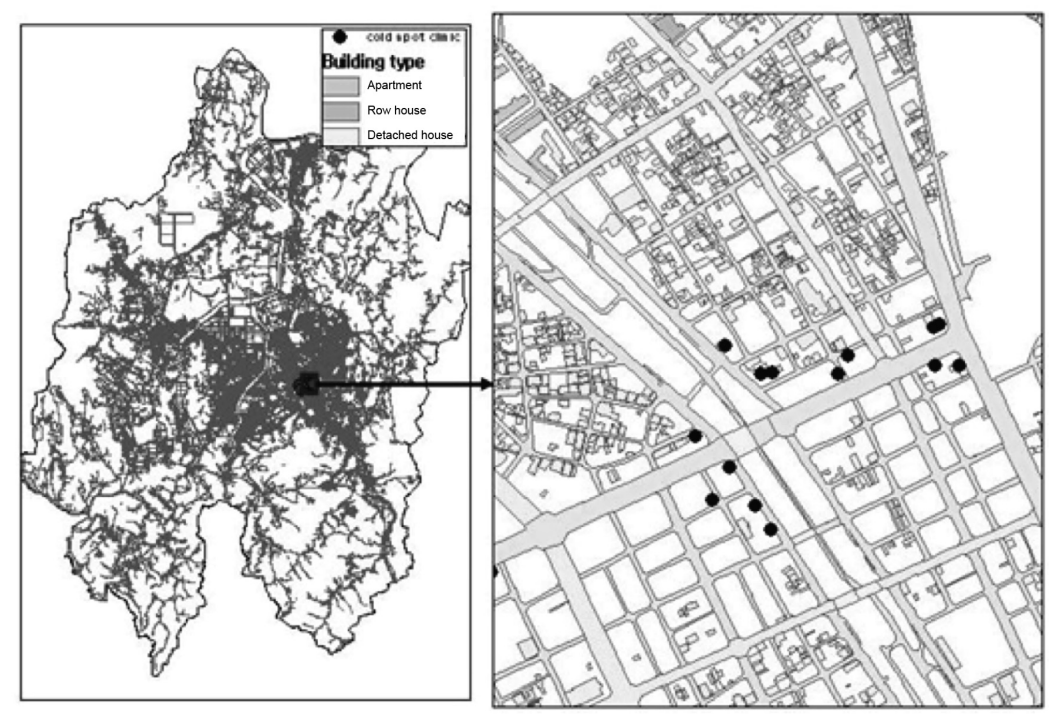

Fig. 3. Cold-spot area for clinics in Daejeon, South Korea. 
Table 3. Location of clinic with respect to specialty in Daejeon, South Korea.

\begin{tabular}{|c|c|c|c|c|c|c|c|}
\hline & \multicolumn{2}{|c|}{ Cold spot } & \multicolumn{2}{|c|}{ Hot spot } & \multicolumn{2}{|c|}{ Other } & \multirow{2}{*}{ Total N } \\
\hline & $\mathrm{N}$ & $(\%)$ & $\mathrm{N}$ & $(\%)$ & $\mathrm{N}$ & $(\%)$ & \\
\hline Family medicine & 0 & $(0.0)$ & 9 & $(16.1)$ & 47 & $(83.9)$ & 56 \\
\hline Internal medicine & 1 & $(0.6)$ & 27 & $(17.2)$ & 130 & $(82.2)$ & 158 \\
\hline Anaesthesiology & 0 & $(0.0)$ & 11 & $(30.6)$ & 25 & $(69.4)$ & 36 \\
\hline Pathology & 0 & $(0.0)$ & 2 & $(100.0)$ & 0 & $(0.0)$ & 2 \\
\hline Urology & 4 & $(8.9)$ & 13 & $(28.9)$ & 28 & $(62.2)$ & 45 \\
\hline Obstetrics and gynaecology & 1 & $(1.3)$ & 20 & $(25.3)$ & 58 & $(73.4)$ & 79 \\
\hline Plastic surgery & 0 & $(0.0)$ & 19 & $(73.1)$ & 7 & $(26.9)$ & 26 \\
\hline Paediatrics & 0 & $(0.0)$ & 7 & (9.1) & 70 & $(90.9)$ & 77 \\
\hline Neurology & 0 & $(0.0)$ & 3 & $(27.3)$ & 8 & $(72.7)$ & 11 \\
\hline Neurosurgery & 0 & $(0.0)$ & 5 & $(50.0)$ & 5 & $(50.0)$ & 10 \\
\hline Ophthalmology & 1 & $(2.5)$ & 12 & $(30.0)$ & 27 & $(67.5)$ & 40 \\
\hline Radiology & 0 & $(0.0)$ & 1 & (9.1) & 10 & $(90.9)$ & 11 \\
\hline General surgery & 1 & $(2.1)$ & 11 & $(23.4)$ & 35 & $(74.5)$ & 47 \\
\hline Otorhinolaryngology & 3 & $(4.8)$ & 10 & $(16.1)$ & 49 & $(79.0)$ & 62 \\
\hline Rehabilitation medicine & 0 & $(0.0)$ & 7 & $(28.0)$ & 18 & $(72.0)$ & 25 \\
\hline Neuropsychiatry & 3 & $(6.7)$ & 11 & $(24.4)$ & 31 & $(68.9)$ & 45 \\
\hline Orthopaedics & 0 & $(0.0)$ & 7 & $(9.3)$ & 68 & $(90.7)$ & 75 \\
\hline Laboratory medicine & 0 & $(0.0)$ & 0 & $(0.0)$ & 2 & $(100.0)$ & 2 \\
\hline Dermatology & 1 & $(3.3)$ & 15 & $(50.0)$ & 14 & $(46.7)$ & 30 \\
\hline Thoracic/cardiovascular surgery & 0 & $(0.0)$ & 2 & $(20.0)$ & 8 & $(80.0)$ & 10 \\
\hline No indication of specialty & 3 & $(2.2)$ & 12 & $(9.0)$ & 119 & $(88.8)$ & 134 \\
\hline Total & 18 & $(1.8)$ & 204 & $(20.8)$ & 759 & $(77.4)$ & 981 \\
\hline
\end{tabular}

explaining the number of clinics. The number of businesses produced a larger regression coefficient $(\mathrm{B}=0.66, \mathrm{P}<0.05)$ than did the population over 65 years $(\mathrm{B}=0.37, \mathrm{P}<0.05)$ and the number of employ- ees $(\mathrm{B}=0.23, \mathrm{P}<0.05)$. In model 3 , the number of employees was a significant predictor of the clinic number $(\mathrm{B}=2.43, \mathrm{P}<0.01)$. No variable in model 2 was significant.

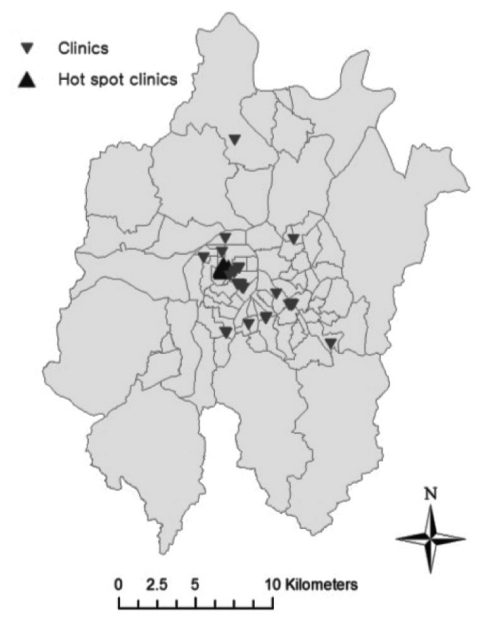

(A) Hot spots for plastic surgery and dermatology

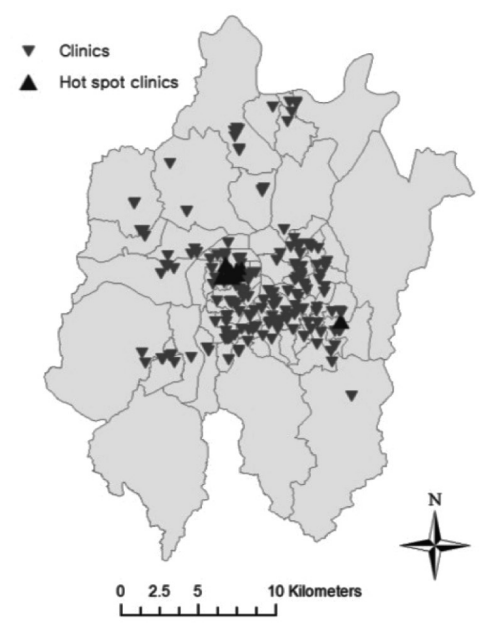

(B) Hot spots for internal medicine and family medicine

Fig. 4. Hot spots by clinic specialty in Daejeon, South Korea. 
Table 4. Regression coefficients of the generalised linear model.

\begin{tabular}{|c|c|c|c|c|c|c|c|c|}
\hline \multirow{2}{*}{ Variable } & \multirow{2}{*}{ Average } & \multirow{2}{*}{ (SD) } & \multicolumn{2}{|c|}{ Model 1} & \multicolumn{2}{|c|}{ Model 2} & \multicolumn{2}{|c|}{ Model 3} \\
\hline & & & B & $\operatorname{Exp}(B)$ & B & $\operatorname{Exp}(B)$ & B & $\operatorname{Exp}(B)$ \\
\hline Total population & 19,417 & $(8,983)$ & 0.00 & 1.00 & 0.00 & 1.00 & 0.00 & 1.00 \\
\hline $\log$ (population $>65$ years) & 1,823 & (943) & 0.37 & $1.45^{*}$ & 0.19 & 1.21 & 0.62 & 1.86 \\
\hline Log (number of business) & 1,188 & (683) & 0.66 & $1.94^{*}$ & 0.24 & 1.27 & -0.47 & 0.62 \\
\hline Log (number of employees) & 5,579 & $(5,226)$ & 0.23 & $1.26^{*}$ & 0.34 & 1.40 & 2.43 & $11.39 *$ \\
\hline $\log \left(\right.$ road area in $\left.\mathrm{m}^{2}\right)$ & 120,393 & $(109,993)$ & 0.00 & 1.00 & 0.00 & 1.00 & 0.00 & 1.00 \\
\hline Deviance/df & & & \multicolumn{2}{|c|}{1.89} & \multicolumn{2}{|c|}{1.01} & \multicolumn{2}{|c|}{0.79} \\
\hline Pearson chi-square/df & & & \multicolumn{2}{|c|}{1.78} & \multicolumn{2}{|c|}{0.91} & \multicolumn{2}{|c|}{1.2} \\
\hline Log likelihood & & & \multicolumn{2}{|c|}{-210.33} & \multicolumn{2}{|c|}{-134.29} & \multicolumn{2}{|c|}{-60.23} \\
\hline
\end{tabular}

*P $<0.05$; model 1 includes all sample clinics with the Poisson regression model; model 2 includes clinics majoring in family medicine and internal medicine with the Poisson regression model; model 3 includes clinics majoring in plastic surgery and dermatology with the negative binomial model.

\section{Discussion}

Convenient road access is considered one of the major factors determining the location of clinics (Vahidnia et al., 2009). The buffer analysis indicated that the clinics were preferentially located along major roads, the $50 \mathrm{~m}$ buffer including most clinic points on the map. This spatial relation has to do with the fact that most people in South Korea do not make reservations for healthcare but arrive at the clinics without prior appointment. Therefore, location along a road advertises the clinic to those on the road granting an advantage in attracting patients.

Previous studies have indicated an imbalance of healthcare resources at the macro level, including the national level, as well as between urban and rural areas in South Korea (Kim, 1995). The hot-spot analysis demonstrated that the density of clinics varied at the city level, thus providing results indicating an imbalance of primary care resources at the lower levels as well. In addition, when testing for differences in distribution by specialty, it was found that relatively few internal medicine and family medicine clinics were located in hot spots. These clinics did not show a high density in specific areas of the city like the clinics specialising in plastic surgery and dermatology did.

These results indicate differences in the policy of locating clinics. A possible explanation for the differences is that there are variations in the number of clinics offering a certain specialty. For example, the number of family medicine and internal medicine clinics was found to be higher than the number of clinics specialising in plastic surgery and dermatology. This may have influenced physicians to open clinics to foster a better distribution by lowering the spatial disparity. A second possibility is that the South Korean health insurance system may have an influence on location due to their system for reimbursement of patient cost. This reasoning would be based on the fact that clinics specialising in dermatology and plastic surgery, which offer many medical services not covered by the National Health Insurance programme, may opt to be located where the local population is commercially active and where the flow of people is high. The hot spots in Fig. 3 and Fig. 5 show a high density of these clinics in areas close to a new section of the city where the population is commercially active or where there are large modern residential complexes. The hot-spot analysis also indicates that some people, e.g. those living outside the city centre, would have to travel further to visit these clinics. Indeed, it has been established that the distance between primary care clinics and potential users influences the use of services (Kinman, 1999; Epstein, 2001; Maheswaran et al., 2006; Tanser et al., 2006). The requirement to travel longer distances is likely to have a negative impact on the use of primary care services but further studies are needed to estimate the effects of travel distances on the use of health care services within a city.

The results of the Poisson regression and the negative Binomial regression models showed that clinic locations were linked to environmental factors in specific areas of the city. Proxy variables for economic status were significant in explaining the variation in the number of clinics in the different parts of the city (but differed depending on the specialties offered). For 
the variables tested in model 1 , the number of businesses produced a larger regression coefficient than did the population over 65 years and the number of employees, which implies that business factors have a stronger impact than the population factor on the total number of clinics operating in a particular dong. These results are in contrast to the idea that the age factor should have a high influence when deciding where to locate clinics even if people over 65 years on average use medical services 2.5 times more than other age groups (Yang, 2006).

No variable in model 2 contributed significantly to an explanation of the variance in to the number of clinics in the different dongs suggesting that the distribution of clinics specialising in internal medicine and family medicine is insensitive to the variance of the independent variables included in this model. In the hot-spot analysis, clinics specialising in internal medicine and family medicine were not densely located in particular areas of the city, a result that was supported by the regression model 2. In model 3, the number of employees was significantly associated with clinic distribution. This variable, used as a proxy variable to represent the economic status of a dong, implies that economic factors are major determinants of the location of these clinics.

The efficient use of healthcare resources is an important issue in health policy and the outcome of this study has major implications for policy makers. Currently, primary care clinics have problems fully performing their role in the healthcare-delivery system, while the uneven spatial distribution of clinics must result in inefficient use of clinic resources. Primary care clinics compete with hospitals to attract patients because patients can freely visit clinics or hospitals without a referral letter (except for tertiary hospitals in Korea). Given the preference for visiting larger healthcare agencies, disparity in the spatial distribution of clinics may have an impact on patient behaviour. People may choose to visit hospitals rather than clinics when they have to travel similar distances, thus leading to increased medical expenses.

The limitations of this study should be noted. First, the study sample included clinics in a metropolitan city. Hence, the study findings cannot be generalised for all levels of the healthcare system, particularly not for the macro level. Second, the classification of clinic specialty was conducted based on the names of clinics, and information regarding specialty was not identified in some clinics. Third, the economic status of people in a dong was measured by two proxy variables rather than by variables representing income level.

\section{Conclusions}

A methodology that can be used for evaluating and understanding how healthcare agencies are distributed is provided. The spatial analysis undertaken has implications for quantitative analysis and evaluation of the arrangement of health resources within a city. Policy makers should prepare plans and policies for restructuring the distribution of health resources.

Different socioeconomic variables are significant in explaining the number of clinics in an area, resulting in inefficient use of healthcare services. No current regulations for the location of clinics exist; policy interventions may be needed to influence the inequity of clinic density at the metropolitan city level.

\section{References}

Alcaraz K, Kreuter M, Bryan R, 2009. Use of GIS to identify optimal settings for cancer prevention and control in African American communities. Prev Med 49, 54-57.

Busato A, Künzi B, 2008. Primary care physician supply and other key determinants of healthcare utilisation: the case of Switzerland. BMC Health Serv Res 11, 8.

Carter RB, Dark FH, Singh AK, 1998. Underwriter reputation, initial returns, and the long-run performance of IPO stocks. J Financ 53, 285-311.

Comber AJ, Brunsdon C, Radburn R, 2011. A spatial analysis of variations in health access: linking geography, socio-economic status and access perceptions. Int J Health Geogr 25, 44.

Crawley MJ, 2007. The R Book. J Wiley \& Son, Chichester, UK. Dulin MF, Ludden TM, Tapp H, Blackwell J, de Hernandez BU, Smith HA, Furuseth OJ, 2010. Using geographic information systems (GIS) to understand a community's primary care needs. J Am Board Fam Med 23, 13-21.

Epstein A, 2001. The role of public clinics in preventable hospitalizations among vulnerable populations. Health Serv Res 36, 405-420.

Getis A, Ord J, 1992. The analysis of spatial association by use of distance statistics. Geogr Anal 24, 189-206.

Gravelle H, Sutton M, 2001. Inequality in the geographical distribution of general practitioners in England and Wales 19741995. J Health Serv Res Policy 6, 6-13.

Guagliardo M, 2004. Spatial accessibility of primary care: concepts, methods and challenges. Int J Health Geogr 26, 3.

Kim YK, 1995. A determinants analysis on regional variation of health care utilization. Yonsei University, doctoral dissertation, 7-13 pp.

Kinman EL, 1999. Evaluating health service equity at a primary care clinic in Chilimarca, Volivia. Soc Sci Med 49, 663-678.

Korean Hospital Association, 2010. 2010 Hospital Annual 
Book, Korean Hospital Association.

Lee KS, Hong SJ, 2010. A study on the relationship between the locational characteristics of Oriental medicine hospitals and the number of patients. Korean J Health Policy Adm 20, 96-112.

Maheswaran R, Pearson T, Jordan H, Black D, 2006. Socioeconomic deprivation, travel distance, location of service, and uptake of breast cancer screening in North Derbyshire, UK. J Epidemiol Commun Health 60, 208-212.

Mantzavinis G, Theodorakis PN, Lionis C, Trell E, 2003. Geographical inequalities in the distribution of general practitioners in Sweden. Lakartidningen 100, 51-52.

Massey F, 1951. The Kolmogorov-Smirnov test for goodness of fit. J Am Stat Assoc 46, 68-78.

McLafferty S, 2003. GIS and health care. Annu Rev Public Health 24, 25-42.

Ministry of Health and Welfare, 2011. Ministry of Health and Welfare Year Book 2011.

Newacheck P, Hughes D, Stoddard J, 1996. Children's access to primary care: differences by race, income, and insurance status. Pediatrics 97, 26-32.
Ord J, Getis A, 1994. Local spatial autocorrelation statistics: distributional issues and an application. Geog Anal 27, 286306.

Parchman ML, 1995. Small area variation analysis: a tool for primary care research. Fam Med 27, 272-276.

Phillips R, Kinman E, Schnitzer P, Lindbloom E, Ewigman B, 2000. Using geographic information systems to understand health care access. Arch Fam Med 9, 971-978.

Tanser F, Gijsbertsen B, Herbst K, 2006. Modeling and understanding primary health care accessibility and utilization in rural South Africa: an exploration using a geographical information system. Soc Sci Med 63, 691-705.

Theodorakis PN, Mantzavinis GD, 2005. Inequalities in the distribution of rural primary care physicians in two remote neighboring prefectures of Greece and Albania. Rural Remote Health 5, 457.

Vahidnia M, Alesheikh A, Alimohammdi A, 2009. Hospital site selection using fuzzy AHP and its derivatives. J Environ Manage 90, 3048-3056.

Yang BM, 2006. Health Economy, Nanam, Seoul, pp. 367-368. 